\title{
50GHz Gallium Arsenide Electro-optic Modulators for Spaceborne Telecommunications
}

Robert Walker, Nigel Cameron, Yi Zhou, Chris Main, Gary Hoy, Stephen Clements

SPIE.

\section{Why GaAs Electro-Optic Modulators?}

The European Space Agency (ESA) has defined electro-optic modulators as a Critical Space Technology" for microwave photonic payloads, with interest in $50^{+} \mathrm{GHz}$ in the ascendant. Of the possible technologies for such devices, GaAs/AlGaAs uniquely benefits from a space-qualified foundry manufacture route, with well proven MMIC-compatible manufacture to industry-standard design-rules.

The material system features:

A fast Linear Electro-Optic (LEO) effect;

AlGaAs unconditionally lattice-matched to GaAs

providing unfettered design potential;

High electron mobility and intrinsic resistivity

Conductivity engineered by epitaxial doping.

GaAs has excellent environmental credentials, is radiation resistant and offers high bias-point stability, so temperature control is not essential. It is a preferred material for solarcells in space and for mm-wave electronics.

\section{Loaded-Line Mach-Zehnder Modulator}

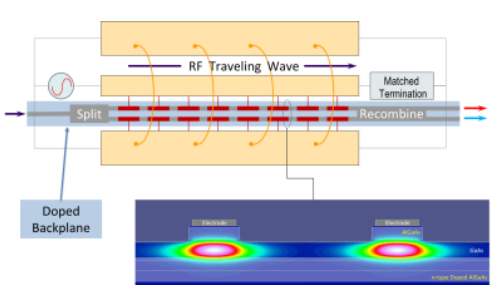

Velocity-matching maintains phase between RF and optical traveling waves over distance; so low drive voltage becomes compatible with high bandwidth.

In GaAs, RF and optical velocities are readily matched using a slow-wave structure, with electro-optic electrode segments arrayed along a coplanar transmission-line.

RF velocity is controlled by the capacitive loading of the electrodes. This is set by the geometry and filling-factor of the electrode array.

RF loss and dispersion are dominant at high frequency; these are accurately modelled and carefully managed to achieve $>50 \mathrm{GHz}$ bandwidths.

\section{Folded Path Modulators \& IQ Arrays}

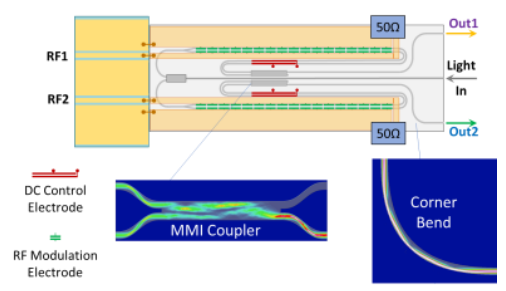

The preferred device configuration is compact, with a straight RF path but convoluted optics. RF \& optical interfaces are segregated to opposite ends, providing:

- High RF feed integrity for maximum bandwidth;

- Superior channel balance in arrays and IQ modulators;

A compact chip, little longer than the RF active zone.

- Minimal package footprint, with the space-hungry fibre interfacing concentrated at one end.

\section{Corner Bends for Path-Folding}

Compact guided-wave structures, especially bends, need a strongly-confining deep-ridge waveguide type.

State-of-art dry-etch processing provides low-loss waveguides with smooth vertical walls within $1^{\circ}$ of vertical. Similar low losses $<0.3 \mathrm{~dB} / \mathrm{cm}$ are measured for both deep and shallow etched waveguide types.
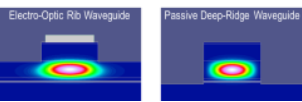

Corner bends use tailored mode-interference to re-image the input to the output. Radius and shaping-factor are critical parameters.

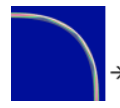

Simulation of $90^{\circ}$ bend

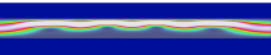

Effective bend radii typically range from 100 to $200 \mu \mathrm{m}$. Measured losses from multi-bend test structures show low losses around $0.015 \mathrm{~dB}$ per bend.

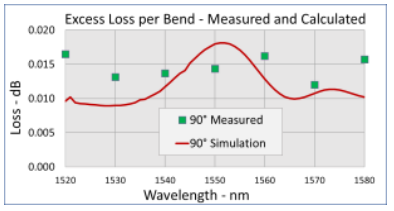

Such low-loss $90^{\circ}$ bends are a critical enabler for the pathfolding which provides a major reduction in module size.

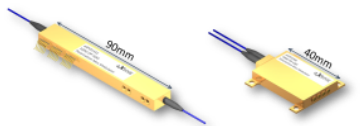

\section{Optical Split \& Recombine}

The preferred MMI coupler type harnesses multi-mode interference in a wide ridge waveguide to re-image inputs to the output plane. MMls offer a compact, split and recombination function.

This is tolerant to manufacturing variability with broadband split balance. They offer low-loss at the design wavelength, but is but the loss increases away from the design-centre.

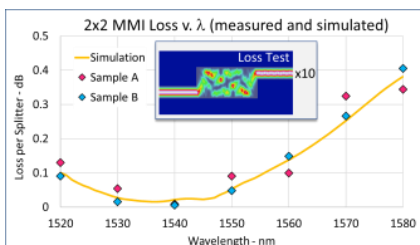

Alternatives such as $Y$-branches, not based on modeinterference, are useful for broadband optics. However, they are less process-tolerant and there is no useable fourth port. Y-branches are typically much longer than MMIs, requiring gradual separation of the waveguides, small bending curvature and a finely defined septum.

\section{Y-branch Simulation}

\section{Measured RF Characteristics}

The design objective was for an electro-optic respons bove $-5 \mathrm{~dB}$ at $50 \mathrm{GHz}$. A V target of $\angle 5 \mathrm{~V}$ allowed for some shortening of the active length, easing RF loss and dispersion constraints, compared with previous $3 \mathrm{~V}$ designs.
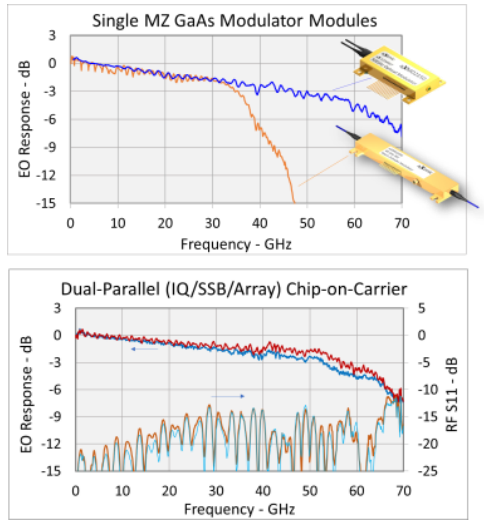

Single MZ modulators and dual-parallel IQ variants have been demonstrated. Both achieve the objective: ○ $\mathrm{V} \pi=4.6 \pm 0.1$

Typical EO response: $-4 \mathrm{~dB}$ at $50 \mathrm{GHz} ;-6 \mathrm{~dB}$ at $65 \mathrm{GHz}$ Packaged $3 \mathrm{~dB}$ bandwidth: $>46 \mathrm{GHz}$

\section{Short Wavelength AIGaAs Modulators}

Space applications are not tied to telecoms bands $1550 \mathrm{~nm}$. Short wavelength (e.g. 1064nm) accesses high solid-state laser power; moreover AlGaAs modulators can provide laser power; moreover AIGaAs modulators can
much lower drive-voltage since at short wavelength: a) EO coefficients (linear and quadratic) rise sharply; b) Refractive index ( $n$ ) rises: EO effects increase as $n^{3}$ c) Electrode is optically longer; $V \pi$ scales directly.

With proper design, a four-fold reduction in drive-power may be possible at $1064 \mathrm{~nm}$ compared with $1550 \mathrm{~nm}$.

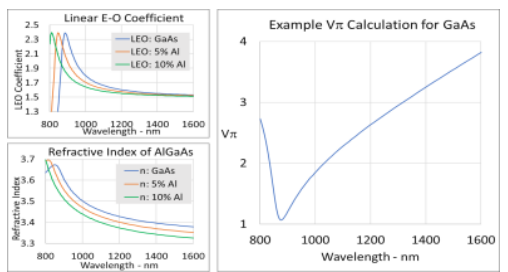

\section{Conclusions}

- Folded GaAs EO modulators have achieved $50 \mathrm{GH}$ bandwidths and $V \pi$ of $4.6 \mathrm{~V}$ at $1550 \mathrm{~nm}$. Single MZM and dual IQ configurations show similar characteristics.

The folded-optics configuration provides a straigh uncompromised RF feed. It halves the fibre handling length and shrinks both chip and module, reducing cost, footprint and weight.

- The RF and optical I/O arrangement enables extension to larger monolithic modulator arrays without requiring a significantly larger package.

- Designs for $1064 \mathrm{~nm}$ (or shorter wavelength) show potential for a fourfold drive-power reduction.

* ESA: Technologies for European Non-Dependence and Competitiveness, SPACE-10-TEC-2018 (Guidance Document for Horizon 2020 Work Programme 2018-2020)

http://ec.europa.eu/research/participants/data/ref/h2020/other/guides_for_applicants/h2020-supp-info-space-10-18-20_en.pdf 